\title{
The influence of number series range, mean, and context upon numerical prediction
}

\author{
ROBERT L. FOWLER \\ University of South Florida, St. Petersburg, Florida 33701
}

\begin{abstract}
Numerical prediction was examined with two-digit number series as stimuli. Three series means (20, 40,80 ) were combined factorially with three series ranges $( \pm 5 \%, \pm 10 \%, \pm 20 \%)$, and subjects' task was to predict future values of the series representing each treatment combination by examining a portion of the series. When subjects were told that the numbers represented the price of an object, reliable within subject differences were produced, with optimism being greatest when series volatility: (range) was highest at two of the three levels of average absolute value (mean). The results were viewed as supporting a prediction by representativeness theory and were consistent with studies employing categorical prediction in which subjects demand larger payoffs when variance is higher.
\end{abstract}

Much of the recent research on human decision making involving risk has been conducted within the context of choices among gambles (Rapoport \& Wallsten, 1972). In the gambling games the optimum strategy, usually defined as accruing the largest possible gain (or the smallest possible loss) in dollars and cents, can always be specified beforehand. Any deviation between a gambler's choices and this optimum strategy can theoretically be analyzed in terms of his preferences for one or more parameters of the gambles presented (Edwards, 1961). One way to characterize such a gamble is in terms of the expected value, variance, and skewness of its probability density distribution over money. In studies where one of these parameters is varied while the others are held constant, preferences have sometimes emerged (Coombs \& Pruitt, 1960; Edwards, 1953) but in other situations have failed to appear (Payne \& Braunstein, 1971; Slovic \& Lichtenstein, 1968).

In laboratory duplex gambling situations (Slovic, 1967), the subject is asked to choose between bets with equal outcomes (expected values) but which differ in parameters such as the probability of winning, probability of losing, amount to win, and amount to lose. Preferences in this case have most clearly developed as a function of the stated probabilities and amounts rather than the moments of the underlying distribution (Payne \& Braunstein, 1971). Kahneman and Tversky (1973) distinguished between this type of category prediction, in which the prediction is in nominal form, and numerical prediction, where the decision is rendered in number form. This latter class of prediction has received little attention as a topic for basic research although many practical decisions are the result of processing a continuous series of value information before accepting a single value as representative or "right," rather than involving only discrete outcome probability functions.

This paper is sponsored by D. E. Clement, who takes full editorial responsibility for it.
It is conceivable that people may possess subjectively based preferences for certain aspects of number series when the numbers are believed to represent the value or price of some object. Analogous to the expected value and variance of the duplex gamble outcomes are the average absolute value (mean) and the volatility (range), respectively, of the available price history information. The present study examined the manner in which these two variables combine to affect preferences for some number series over others. Preference was defined as relative optimism (or pessimism) regarding the future price of an object when the previous prices of that object were represented by a displayed number series. Analyses of economic behavior have shown that the modal investor seeks to avoid risks and requires a higher expected value as "compensation" for higher variance (Bell \& Short, 1971), and studies of psychophysical judgment indicate that the magnitude of the power law exponent obtained in direct scaling methods is related to the range of numbers employed but does not depend upon the modulus of the range (Stevens, 1971). Thus, it was expected that optimism regarding price changes would vary directly with the volatility of the object price history, but would be relatively independent of the mean of previous prices.

\section{METHOD}

\section{Subjects}

The subjects were 110 undergraduate men and women who volunteered to serve, and most received extra credit in courses for which they were registered.

\section{Materials}

The stimuli for this study were number series consisting of 12 two-digit integers drawn randomly from populations having rectangular distributions with means equal to either 20,40 , or 80 and ranges equal to either $\pm 5 \%, \pm 10 \%$, or $\pm 20 \%$ of the population mean. These values were chosen because they are representative of the most probable prices at which human decisions are made in real life. Each of the three means was combined factorially with each of the three ranges to form the 
Table 1

Actual Parameters of the Number Series Stimuli and Observed Statistics for Each Cell of the Design

\begin{tabular}{|c|c|c|c|c|c|c|c|}
\hline \multirow[b]{3}{*}{ Cell } & \multirow{2}{*}{\multicolumn{3}{|c|}{ Actual Parameters }} & \multicolumn{4}{|c|}{ Observed Statistics* } \\
\hline & & & & \multicolumn{2}{|c|}{ Price Context Group } & \multicolumn{2}{|c|}{ Random Context Group } \\
\hline & $\mu$ & $\sigma^{2}$ & Range $\dagger$ & Mean & $\begin{array}{l}\text { Proportion } \\
\text { Optimistic Ss }\end{array}$ & Mean & $\begin{array}{l}\text { Proportion } \\
\text { Optimistic Ss }\end{array}$ \\
\hline 1 & 20.0 & 67 & $19-21$ & 20.4 & .81 & 20.1 & .50 \\
\hline 2 & 20.0 & 2.00 & $18-22$ & 20.2 & .57 & 19.9 & .20 \\
\hline 3 & 20.0 & 6.67 & $16-24$ & 20.6 & .63 & 20.2 & .55 \\
\hline 4 & 40.0 & 2.00 & $38-42$ & 40.2 & .39 & 40.3 & .40 \\
\hline 5 & 40.0 & 6.67 & $36-44$ & 40.5 & .61 & 40.2 & .55 \\
\hline 6 & 40.0 & 24.00 & $32-48$ & 40.4 & .51 & 40.1 & .45 \\
\hline 7 & 80.0 & 6.67 & $76-84$ & 79.9 & .50 & 80.2 & .38 \\
\hline 8 & 80.0 & 24.00 & $72-88$ & 80.1 & .77 & 80.0 & .33 \\
\hline 9 & 80.0 & 90.67 & $64-96$ & 82.0 & .73 & 79.6 & .48 \\
\hline
\end{tabular}

*Based on the mean of four responses per subject.

†Each number within the given range was equally likely to occur.

nine cells of the experimental designs as shown in Table 1. Each subject was exposed to four samples from each cell for a total of 36 series in all.

The number series were arranged for presentation on equidistant horizontal lines, four per page on letter size sheets of paper. The series appearing together on a particular page were systematically combined in four different orders to form nine-page booklets. The order of appearance of pages in the booklets was randomized for each subject.

\section{Procedure}

For one randomly assigned group of subjects $(\mathrm{N}=70)$, called the price context group (Group PC), the cover sheet of the booklet contained instructions which informed the subject that he was to examine each number series separately, assuming that it represented the value of some item in each of 12 different consecutive, and equal time periods. The subject was asked to predict what the value of the item would be at the end of a series exactly twice as long as and including the one shown, i.e., to predict the 24 th price in the series. This was done to simulate a situation in which speculation regarding future price fluctuations would be required in order to make a decision.

Subjects in a second condition $(\mathrm{N}=40)$, referred to as the random context group (Group RC), were exposed to the same series as Group PC but were given a different context for the instructional set. These subjects were shown a large bag containing 300 poker chips, an equal number of which had the numerals 19,20 , or 21 stamped on one side. (This represented the population of numbers for the first cell of the experimental design.) The subject was asked to shake the bag thoroughly and remove 23 chips, one at a time without looking. The first 12 of these chips were then placed in the order drawn, with numerals up, on the table in front of the subject. The subject was then told to examine the entire series and predict what number would be stamped on the next (24th) chip drawn. This was considered a practice trial and the subject was informed that each of the 36 series in the booklet he was about to examine was generated in the same manner. Thus, for these subjects, no mention was made of the fact that the numbers in the series represented the value of some item. The primary purpose of including Group RC in the study was to control for the possible presence of trends in the number series in some cells due to random sampling.

All subjects marked their predictions on an answer sheet and no feedback regarding "correctness" was given. They were instructed to refrain from prolonged consideration of any particular series and to respond with the first number which seemed appropriate. All subjects completed the task within $1 \mathrm{~h}$.

\section{RESULTS}

The mean of each subject's predicted values for each treatment combination was expressed as a percentage of the population mean from which the series of numbers for that cell was drawn and became the basic dependent variable. These data were subsequently evaluated, separately for the price context and random context conditions, as randomized block factorial analyses of variance (Kirk, 1968). For Group PC, the main effects of volatility $[F(2,552)=9.34, p<.001]$, and mean absolute value $[F(2,552)=8.40, p<.001]$, were both highly significant. Optimism was an increasing function of volatility, yielding means of $100.8,101.3$, and 102.1 for the $\pm 5 \%, \pm 10 \%$, and $\pm 20 \%$ volatility conditions, respectively, and a trend analysis based on orthogonal coefficients transformed for the unequal volatility intervals showed that the departure from linearity was not reliably different from chance, $F(1,552)<1$. While this finding lends fiducial support to the hypothesis of higher payoffs being expected (predicted) by subjects in exchange for tolerating (observing) higher variances, the significant interaction of volatility (VTY) and mean absolute value (MAV) $[\mathrm{F}(4,552)=6.09, \mathrm{p}<.001]$, makes the interpretation more complex. That is, the rank order of VTY means was not the same at each level of MAV, as Figure 1 clearly depicts. An analysis of the simple main effects and subsequent applications of Tukey's HSD test for a posteriori pairwise comparisons (Kirk, 1968) showed that the $\pm 20 \%$ VTY condition produced significantly higher $(\mathrm{p}<.01)$ predictions than the two lower VTY values at both the MAV 20 and MAV 80 levels, but not at MAV 40 . The $\pm 5 \%$ VTY resulted in reliably lower $(\mathrm{p}<.01)$ predictions than the other VTY levels at a MAV of 80, and was intermediate in value at a MAV of 20 . Much of the significant interaction appears to be attributable to the nonmonotonicity of the $\pm 20 \%$ VTY level and the relatively flat trend of the $\pm 10 \%$ VTY condition. Thus, 


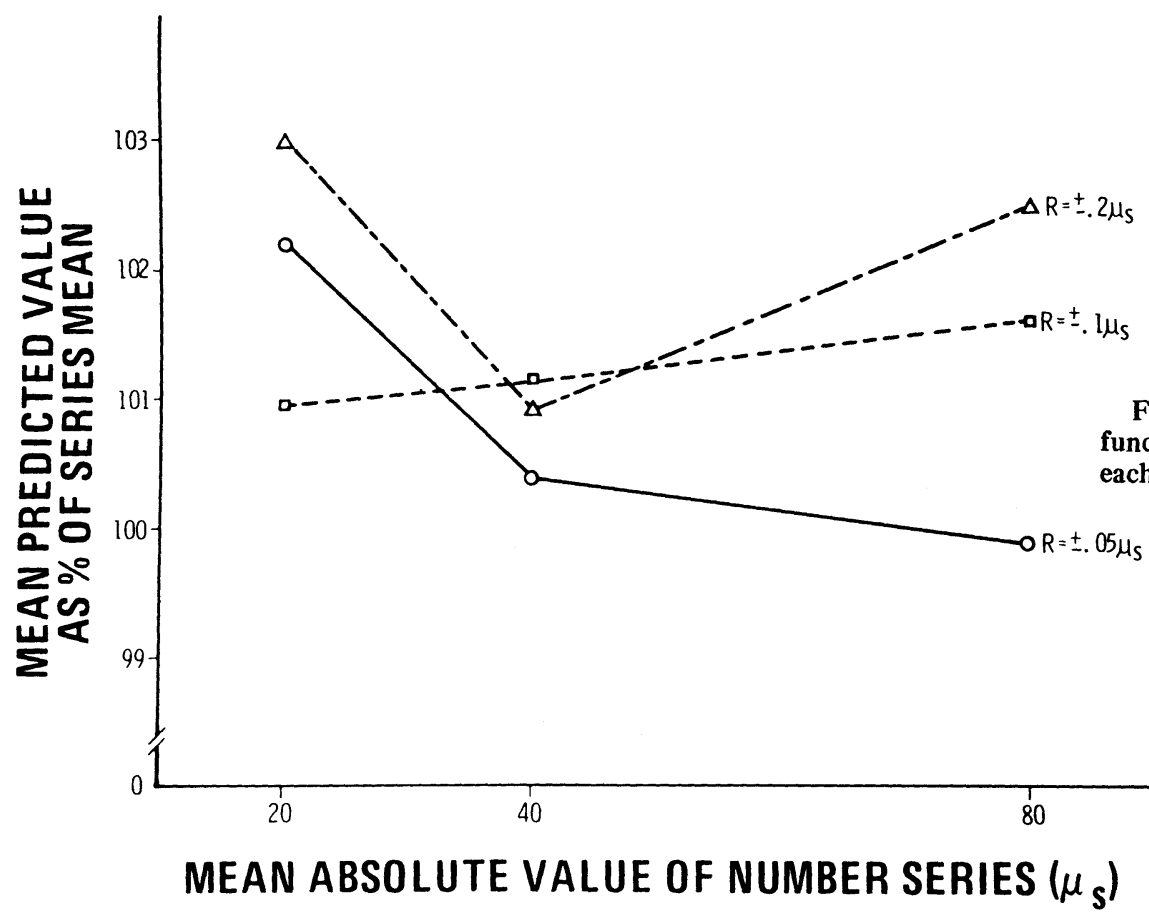

the observed preferences, reflected in differential predictions of future values, appeared to develop at the extremes of both the MAV and the VTY continua, whereas the intermediate values of each produced no differences in predictions.

The marked influence that the combination of price volatility and absolute value has on predicted price was clearly a function of the context in which the predictions were made, for Group RC exhibited no such behavior (all Fs $<1$, dfs $=2 / 312$ and 4/312, for the main effects and the interaction, respectively). As seen in Table 1, the predictions of Group RC were, in general, closer to the cell parameters than those of Group PC. A consistent finding under virtually all combinations of mean value and volatility was the general optimism of the subjects who believed the numbers represented price changes in objects of value. The overall mean for Group PC (101.4) was reliably higher than for Group RC $(100.2),[\mathrm{F}(1 / 108)=12.00, \mathrm{p}<.001]$. The proportion of subjects in each context group whose mean predictions exceeded the normative value for each cell are also given in Table 1.

\section{DISCUSSION}

The findings imply that subjective preferences for numbers may be derived from a person's beliefs about what the numbers represent. Since every number within the range of a series was equally likely to appear among the 12 stimulus numbers shown, predictive uncertainty was maximal. If Group PC had followed the normative theory of prediction, as Group RC apparently did, the mean or the median of the series should have been selected as the 24th price. Whereas only the context describing the meaning of the number series was changed for subjects in the former group, their general level of optimism relative to the normative values, and the highly reliable effects of two basic series parameters upon their demonstrated preferences were quite remarkable. However, these results would follow from the theory of intuitive predictions advanced by Kahneman and Tversky (1973), who propose that people predict by representativeness rather than by prior probabilities. That is, if a person believes that a series of numbers represents the changing prices of an item of value, the statistics of the historical price series are ignored in favor of an outcome based upon what item values are expected to do in the future.

It had been proposed that differential optimism would be evidenced as a function of volatility, with subjects "demanding" higher expected values (future MAVs) in return for tolerating higher variances (volatility). This did happen at the extremes of the MAV distribution, but the significant interaction questions the acceptance of a general hypothesis of compensation. Particularly inconsistent with this hypothesis is the marked optimism when the MAV of 20 was combined with the VTY of $\pm 5 \%$. Two explanations for this finding are offered. First, in order to reflect optimism, the subject had to choose the next highest whole integer, 21 , as a prediction, which is a relative extreme $105 \%$ of the series mean. Whereas a clear majority of subjects in the experimental group (56 out of 70 ) were optimistic overall, it follows that the cell in question would produce higher mean predictions than others in the design because of the normalized scoring procedure. Second, the number 21 has been found to have the 6th highest rated association value of all numbers between 0 and 100 , while the numbers 19 and 20 were ranked 39 th and 19th, respectively (Batting \& Spera, 1962). With the exception of the combination of lowest MAV and smallest VTY, higher volatilities produced greater optimism at both MAVs (20 and 80$)$ where preferences were most evident. This can be interpreted as implying that if the human judge is indifferent about a particular range of prices, then fluctuations within this range will not greatly influence the decision rendered. However, where certain price norms are preferred, previously observed variations around the mean of a preferred price range have marked effects on predictions, and presumably, decisions to buy or sell.

The general tendency of the price context subjects to make 
higher predictions than the random context subjects under all price history conditions may reflect the widely held conviction among the public that prices of most objects increase with the passage of time. Consistent with this explanation is the finding that when students must predict future academic achievement of a fellow student on the basis of imperfect information, they exhibit leniencey; i.e., they respond to a reduction of validity by raising the predicted level of performance (Kahneman \& Tversky, 1973). Such supranormative estimates may be a general characteristic of the human judge when numerical predictions are rendered under uncertainty. Labeling the phenomenon "optimism" or "leniency" would depend upon the particular context involved rather than indicating different underlying processes. More importantly, within the context of item prices, the degree of this inflation philosophy can apparently be augmented, or diminished by changing the parameters of the historical numerical information supplied to the judge. This also implies that the choice of range and modulus in studies of numerical prediction in other contexts should be carefully considered, particularly in view of the potentially complex interactive effect these parameters may have upon behavior.

\section{REFERENCES}

Battig, W. F., \& Spera, A. J. Rated association values of numbers from 0-100. Journal of Verbal Learning and Verbal Behavior,
$1962,1,200-202$.

Bell, D. \& Short, J. Investment selection as a function of the variance of previous payoffs. Experimental Publication System, 1971, 10, Ms.No. 394-12.

Coombs, C. H., \& Pruitt, D. G. Components of risk in decision making: probability and variance preferences. Journal of Experimental Psychology, 1960, 60, 265-277.

Edwards, W. Probability preferences in gambling. American Journal of Psychology, 1953, 66, 349-364.

Edwards, W. Behavioral decision theory. Annual Review of Psychology, 1961, 12, 473-498.

Kahneman, D., \& Tversky, A. On the psychology of prediction. Psychological Review, 1973, 80, 237-251.

Kirk, R. E. Experimental design: Procedures for the behavioral sciences. Belmont, Cal: Brooks/Cole Publishing Company, 1968.

Payne, J. W., \& Braunstein, M. L. Preferences among gambles with equal underlying distributions. Journal of Experimental Psychology, 1971, 87, 13-18.

Rapoport, A., \& Wallsten, T. S. Individual decision behavior. Annual Review of Psychology, 1972, 23, 131-176.

Slovic, $P$. The relative influence of probabilities and payoffs upon perceived risk of a gamble. Psychonomic Science, 1967, 9, 223-224.

Slovic, P., \& Lichtenstein, S. Importance of variance preferences in gambling decisions. Journal of Experimental Psychology, 1968, 78, 646-654.

Stevens, S. S. Issues in psychophysical measurement. Psychological Review, 1971, 78, 426-450.

(Received for publication September 9, 1974.) 